Journal of Social Sciences 5(1): 60-66, 2009

ISSN 1549-3652

(C) 2009 Science Publications

\title{
Analysis of the Relationship Between Physical Self-Concept and Body Image Dissatisfaction in Female Students
}

\author{
${ }^{1}$ Sirus Alipoor, ${ }^{1}$ Ali Moazami Goodarzi, ${ }^{2}$ Maryam Zarra Nezhad and ${ }^{3}$ Lale Zaheri \\ ${ }^{1}$ Department of Psychology, Faculty of Education and Psychology, \\ Shahid Chamran University, Ahvaz, Iran \\ ${ }^{2}$ Islamic Azad University of Ahvaz, Ahvaz, Iran \\ ${ }^{3}$ National Iranian Oil Company Hospital, Ahvaz, Iran
}

\begin{abstract}
Problem statement: Body image is a psychological construct which refers to self-concept including self image and feelings an individual perceives about his or her body. Body image can significantly affect the way an individual perceives about his or her body. Studies on relationship between physical self-concept and body image in some countries showed that the women's body dissatisfaction has led to increase in eating disorders. The question was raised whether such a relationship may exist in Iran. Approach: The purpose of this study was to investigate the relationship between physical self-concept and body Image dissatisfaction in female. The importance of body image in Iranian culture has, recently, became more significant to the extent that tremendous expenditure of time, effort and money has been paid by people, particularly by women, seeking to alert their appearances to resemble an idea image. A survey of 140 female students selected through random sampling was conducted in Shahid Chamran University of Ahvaz, southern Iran. To collect data, a questionnaire including Demographic Information form the Physical Self-Perception Profile (PSPP) and Nine Figure Silhouette Scale was used. The physical self-perception profile have five subscales, which were sport competence (SPORT), body attractiveness (BODY), physical condition (CONDITION) and physical strength (STRENGTH) and one subscale that assesses a global perception of overall Physical Self-Worth (PSW). Results: The finding of the research indicated that there is a significant negative correlation between the physical self-concept and body image dissatisfaction. The correlations magnitude ranged between $0.49-0.79$ for body image dissatisfaction. Body image dissatisfaction correlated strongest with physical self-worth and with body physical self-concept in female students in Iran. Thinness had the most influential impact on body image dissatisfaction. The findings also showed discrepancy between the ideal and current figures. The participations presented an ideal body that was much thinner than their perceived size. Conclusion/Recommendation: Women most at risk of body dissatisfaction are those who have low self-concept, those who perceive a lack of control over their bodies and those who have internalized the thin ideal. Exposure to particular kinds of television images may influence body satisfaction in females. The content of television images affects body satisfaction and drive for thinness in females. High self-concept, resistance to internalization of the thin ideal and perceptions of body mastery and control predict body satisfaction. Moderate exercise, focusing on body mastery and enjoyment rather than appearance and weight, may also be an effective strategy for improving body satisfaction. Cultural changes and a focus on body function rather than aesthetics would be likely to improve body satisfaction.
\end{abstract}

Key words: Physical self-concept, body image, body dissatisfaction, ideal body and self-perception

\section{INTRODUCTION}

Body image is a psychological construct which refers to self-concept including self image and feelings an individual perceives about his or her body. Body image can significantly affect the way an individual perceives and interacts with the surrounding environment. An individual with a distorted body image may also suffer from low self-concept or lack feelings of self-worth, based on perceived physical appearance. The construct of body image can be broken down two and often three dimensions. The first dimension is

Corresponding Author: Sirus Alipoor, Department of Psychology, Faculty of Education and Psychology,

Shahid Chamran University, Ahvaz, Iran 
cognitive, how an individual "thinks" he or she looks. The second is emotional, how an individual "feels" he or she looks. Last is idealistic, how an individual "wants" to look ${ }^{[1]}$. All three dimensions can different at any given point in time. In Iranian culture, body image has, recently, became more significant. Many people especially women spend tremendous expenditure of time, effort and money to alert their appearances to resemble an idea image. The term body image has numerous connotations associated with it particularly for females. Females are more likely than male to struggle with body image distortion mainly due to societal messages which are often conflicting and confusing regarding female roles and expectations. Because of this analysis of the relationship between Physical self-concept and body Image dissatisfaction is of great importance.

There are various social influences affecting individuals body image at any point during the life cycle, such as parents, media and friends ${ }^{[2,3]}$. Contemporary North American Society tends to tell a stereotypical view of the attractive women as one who is "firm but shapely, fit but sexy, strong but thin ${ }^{[4] \text {. }}$

Psychological models of body image have shown significant development in the last 10 years. In 2002, Thomas Cash ${ }^{[5,6]}$ presented a cognitive-behavioral model of body image development and experiences that emphasized the importance of cultural socialization, interpersonal characteristics, physical characteristics and personality attributes in body image evaluation and investment. This model recognizes the reciprocal relationship between environmental events; cognitive, affective and physical processes; and individual's behaviors in determining body image and has been influential in the last few years.

Self-perceptions influence the individual's behavior and in turn the way that the individual perceives him or herself. Self-concept is important as an outcome and as a mediating variable that helps to explain other outcome $^{[7]}$.

The sociology of the body become an established discipline in the $1990 \mathrm{~s}$, Bryan Turner $^{[8]}$ coining the term "somatic society" to describe the newfound importance of the body in contemporary sociology.

Feingold and Mazzella ${ }^{[9]}$ conducted a meta analysis of gender differences in attractiveness and body image using 222 studies from the past 50 years. The findings of the research showed that the prevalence of eating disorders in women has risen because of increases in women's body dissatisfaction. The findings also showed dramatic increases in the numbers of women among individuals who have poor body image. These trends were found across multiple conceptualizations of body image, including selfjudgments of physical attractiveness.

Hagger et $a l .^{[10]}$ examined the generalizability of the form, structural parameters and latent means of a hierarchical multidimensional model of physical selfperceptions in adolescents from different cultures including British, Hong Kong and Russian. The findings of the research showed that tests of crosscultural generalizability of the proposed model supported the invariance. Canpolat et al. ${ }^{[11]}$ examined the roles of body image, ideal body weight, selfperception and Body Mass Index (BMI) on the dieting behavior of Turkish adolescents. The sample $(n=531)$ of the study ranged from 15-17 years old students from five selected high schools in Ankara. The SelfPerception Profile (SPPA), the Body Image Satisfaction Questionnaire (BISQ) and the Dieting Status Measure (DiSM) were conducted. The results showed that dieting adolescents received significantly lower scores than non-dieters for most of the BISQ items and for the physical appearance and global self-worth subscales of the SPPA. The findings also showed that a thinner body ideal, low self-worth and low physical self-concept have more significant effects on body dissatisfaction and dieting than being actually overweight does. Furthermore, High physical self-concept scores and body satisfaction may not necessarily preclude having a thinner body ideal and, hence, dieting in girls.

Anderson ${ }^{[12]}$ found that girls and women who have higher levels of exposure to media imagery tend to be less satisfied with the way that they look than those with lower levels of exposure. Tiggemann and Golder $^{[13]}$ recently ran a prospective study of body image and media exposure with 214 Australian girls (mean age 14 years). At time1, they completed questionnaire measured of media exposure, internalization of appearance ideals, appearance schemas, body dissatisfaction and drive for thinness. One year later, at time 2 , they completed the same measured. Tiggemann and Golder ${ }^{[13]}$ found that none of the media exposure measures at time 1 predicted body image at time 2. She also found that body image at time 1 did not predict change in media exposure. People higher in self-esteem in general tend to be more satisfied with their bodies.

Paxton $^{[14]}$ studied the relation between self-esteem and body image in adolescent. He found that for adolescent girls body dissatisfaction may precede low self-esteem. In the same studies, Connors and Casey ${ }^{[15]}$ indicated that there is a closer association of these two variables for women than for men. In another study, Grogan et $a l .{ }^{[16]}$ found that positive body image has been linked with positive feelings about the self and 


\section{J. Social Sci., 5(1): 60-66, 2009}

feeling of self-concept and power in social situations. Although most authors have assumed that high selfesteem leads to high body satisfaction.

With many studies looking at the self-concept and body image dissatisfaction, more work is needed in this area to determine the effectiveness of programs that aim to build physical self-concept in order to decrease body dissatisfaction. Though some attempts have been made, very little research on the subject has been done in Iran. The main objective of this research is to analyze the relationship between physical self-concept and body image dissatisfaction in female student. This will help build physical self-concept to decrease body dissatisfaction. The main research question is whether there is relationship between physical self-concept and body image dissatisfaction. To answer this question, the following hypotheses (primary and secondary) are proposed:

Hypotheses 1: Five subscales of physical self-concept would be negatively correlated with body image dissatisfaction among female students.

Hypotheses 2: There is a reliable tendency in females to pick a thinner ideal body image than their current figure.

In the present study the term Physical Self-Concept (PSC) defined as a person's perceptions of herself formed through experience with and interpretations of her environment related to her physical domain ${ }^{[17]}$ PSC is linked to Body Image Dissatisfaction (BID). Body image dissatisfaction is the subjective difference between a female current body image (i.e., her mental representation of her body) and her ideal body image.

\section{MATERIALS AND METHODS}

Body image is conceptualized as subjective. There is no simple link between people's subjective experience of their bodies and what is perceived by the outside observer. This is obvious in distortion of body size (e.g., many young women who experience anorexia nervosa believe they are much heavier than they appear and some highly muscled bodybuilders believe that they are less muscular than they are in reality) and in cases of "phantom limb" phenomena (in which people who have had limbs amputated report still feeling the missing limb). It is also relevant to the large number of women and girls who "feel fat" although they are objectively of average (or below average) weight for their height.

Promotion of positive body image is important in improving people's quality of life and physical health, physical self-concept and body image is implicated in a number of health-related behaviors. Psychologists and sociologists have used various measures to assess body image. Many of these techniques were originally produced to assess body dissatisfaction in women who have problematic relations with food. Figural rating scales, or silhouette techniques, were developed in the1950s and remain a widely used quantitative measure of degree and direction of body dissatisfaction. Since body image dissatisfaction have been found to be an important factor for low self-concept, Psychologists and sociologists have used various instruments that measure an individual's physical self- concept.

The research population is the female students of Shahid Chamran University of Ahvaz. Considering the size of population and necessity of representativeness of the sample, the initial size of the sample, using Morgan's formula was 100. The studied sample size which was obtained through random selection increased to 140 . The majority of participants were within the age range of 19-30 in 2008. In order to collect the data, each student was given the Demographic Information form, Physical Self-perception Profile (PSPP) and Nine Figure Silhouette Scale.

Information regarding the participants' date of birth, height and weight was collected by Demographic Information forms

Fox and Corbin ${ }^{[18]}$ have introduced and developed a multidimensional 30-item self report instrument as Physical Self-perception Profile (PSPP) to measure an individual's physical self-concept. It features four domain-specific subscales assessing perceived sport competence (SPORT), body attractiveness (BODY), physical condition (CONDITION) and physical strength (STRENGTH) and one subscale that assess a global perception of overall physical self-worth (PSW). A four-choice structured alternative item format is used with six items per subscale. The subject is first asked which kind of person best describes them (e.g., "Some people always have a really positive feeling about the physical side of themselves" but "Others sometimes do not feel positive about the physical side of themselves") and then to decide to what degree they are that kind of person (e.g., "Sort of true of me" or "Really true of me"). The result is a four choice response. The PSPP was administered to participants in this study by the researcher during a scheduled practice Scoring the PSPP involves adding the scores of each subscale. Each response is assigned a value of 1-4 points. Each subscale, SPORT, CONDITION, BODY, STRENGTH and PSW, contains six items ranging from 6-24. The negative items are reversed so that the lowest-scoring descriptor is placed first and items from each of the 
subdomains are placed in sequence within the complete profile. Therefore, high scores reflect high self-concept. All the subscales have three positive and three negative items except for the STRENGTH subscale which contains four positive and two negative items. Testretest findings for this instrument have been reported by Fox $^{[21]}$ with test-retest reliabilities ranging from 0.74 0.92 over a 16 days period and $0.81-0.87$ over a 23 days period. Two independent samples reported internal consistency reliability using Cronbach's alpha, with a range of 0.81-0.92. Contributions of items to internal consistency were indicated by the correlation of the item to scale total (after elimination of the item). They ranged between 0.5 and 0.7 with a mean corrected itemtotal correlation score for all subscales of 0.69 for females. All items contributed consistently well to the functioning of their subscale.

The Nine Figure Silhouette Scale ${ }^{[19]}$ is one of many silhouette continuums used to measure body satisfaction $^{[20]}$. The scale has 9 female silhouettes evenly distributed across the ectomorphic-endomorphic continuum. Each silhouette is numbered with the smallest ectomorph labeled " 1 " and the largest endomorph labeled "9." Subjects are instructed to indicate a silhouette that best represents their current body size (the mental representation of their body) and then indicate a silhouette that represents their ideal body size. The survey was administered to the participants by the researcher during a scheduled practice. For this study the subjects were asked to identify the body ideal for success in their sport. Calculating the absolute value of the difference between the subject's current body size and the subject's ideal body size produces a positive score. For example, if subject one indicated her current body size as a " 5 " and her ideal body size as a " 3 ," her score would be " 2 ". If subject two indicated her current body size as a " 3 " and her ideal body size as a "5," her score would be the absolute value of " -2 ", equaling " 2 " and her body dissatisfaction would be equal to subject one. Larger scores indicate greater body dissatisfaction. Researchers have established good instrument validity comparing results of silhouette ratings to other body satisfaction scales such as the Body Cathexis Scale, Body Esteem Scale and Body Dissatisfaction Scale of the Eating Disorders Inventory ${ }^{[21-24]}$.

The collected data was imported in to Scientific Package of Social Sciences (SPSS) software. The data were analyzed using correlation statistics. In this study Internal consistency reliability using Cronbach's alpha, for Physical Self-perseption Profile was 0.86 and for Nine Figure Silhouette Scale was 0.93 indicating high level of internal consistency.

\section{RESULTS}

Prior to testing the study's hypotheses, descriptive statistics were computed (mean and standard deviation) to assure that inferential assumptions were met. These are shown in Table 1. According to measurements, high scores in Physical Self-perception Profile reflect high self-concept and larger scores in Nine Figure Silhouette Scale indicate greater body dissatisfaction.

As shown in Table 1, results showed moderate mean values, revealing no noticeable response bias. The main research question was whether there is a statically significant relationship between the five subscales of physical self-concept and body image dissatisfaction among female students. Pearson Product-Moment Correlations (PPMC) were computed to answer this question. The PPMCs of the five subscales of physical self-concept and body image dissatisfaction of female students are shown in Table 2.

Results indicated a significant (0.01 or 0.05) negative moderate correlation between the five subscales of physical self-concept and body image dissatisfaction. The correlations magnitude ranged between 0.49-0.79 for body image dissatisfaction. Body image dissatisfaction correlated strongest with "physical self-worth" and with "body" physical selfconcept.

The results also show that thinness had the most influential impact on body image dissatisfaction. The discrepancy between the two figures (ideal and current) is seen as an indication of dissatisfaction and the figures

Table 1: Descriptive statistics for the variables in this study

\begin{tabular}{lccc}
\hline Variable & Scale's Range & Mean & SD \\
\hline Physical self-Concept & & & \\
Sport & $8-22$ & 14.34 & 3.945 \\
Conditioning & $9-22$ & 16.19 & 3.122 \\
Body & $9-22$ & 16.13 & 2.648 \\
Strength & $8-22$ & 15.50 & 3.467 \\
Physical & & & \\
self-worth & $8-23$ & 16.16 & 3.851 \\
Body image & & & \\
satisfaction & $0-5$ & 0.80 & 0.820 \\
High & $42-78$ & 54.86 & 6.530 \\
Weight & $143-176$ & 161.33 & 5.410 \\
Age & $19-30$ & 21.46 & 2.360 \\
\hline
\end{tabular}

Table 2: Correlations between physical self-concept subscales and body image dissatisfaction in female students

\begin{tabular}{ll}
\hline Physical self-concept & Body image dissatisfaction \\
\hline Sport & $-0.68^{* *}$ \\
Conditioning & $-0.49^{*}$ \\
Body & $-0.61^{* *}$ \\
Strength & $-0.50^{*}$ \\
Physical self-worth & $-0.79^{* *}$ \\
\hline$*: \mathrm{p}<0.05 ; * * \mathrm{p}<0.01$ &
\end{tabular}




\section{J. Social Sci., 5(1): 60-66, 2009}

chosen indicate whether their ideal is thinner or fatter than their current body type. The participations presented an ideal body that was much thinner than their perceived size.

\section{DISCUSSION}

Since in this study the high correlation has been found between, five subscales of physical self-concept and body image dissatisfaction, thus hypothesis 1 holding that, five subscales of physical self-concept would be negatively correlated with body image dissatisfaction among female students, is accepted.

As results showed ideal body was much thinner than perceived size. This means that the respondents wished to be slimmer. Studies using this technique have found that women show a reliable tendency to pick a thinner ideal than their current figure. This effect has been replicated in the USA. Australia and Britain ${ }^{[25]}$. Hence according to the findings, hypothesis 2 holding that there is a reliable tendency in females to pick a thinner ideal body image than their current figure, is accepted.

This finding is precisely in line with previous related studies, mainly after 1990 s, which have been reviewed in the study. Since 1950, researchers have taken "body image" to mean many different things. Kevin Thompson and colleagues ${ }^{[26]}$ in 1999 noted 16 different definitions of "body image" used by researchers and clinicians. These included weight satisfaction, size perception accuracy, appearance satisfaction, body satisfaction, appearance evaluation, appearance orientation, body concern, body esteem, body schema and body percept. The results suggest that there is a negative correlation between the physical selfconcept and body image dissatisfaction.

\section{CONCLUSION}

Researchers have used a variety of different techniques to study how women evaluate their bodies and have concluded that many women in Western culture are dissatisfied with some aspect of their body weight and shape and are taking behavioral steps to try to change the look of their bodies. Psychologists and sociologists have tried to make sense of women's dissatisfaction with body shape, weight and selfperception within a feminist framework. The result is a comprehensive, wide-ranging, but necessarily partial review of the variety of influences on women's body image and the behavioral effects of these influences.

People most at risk of body dissatisfaction are those who have low self-concept, those who perceive a lack of control over their bodies and those who have internalized the thin ideal. Media representations of the slender ideal may lead to unfavorable social comparisons and may result in dissatisfaction, particularly in women who have internalized societal ideals. However, interview work suggests that women in particular are cynical about media portrayal of the "ideal body," and want to see more realistic images of women in the media. Media role models may differ depending on the age of the viewer, with viewers making active choices as to comparison groups.

Mean age of this study was 21.46. The sample hasn't entered to critical stage of aging and gaining weight. Results also showed moderate mean values and less body image dissatisfaction. But there was a reliable tendency to pick a thinner ideal body image than their current figure.

In affluent Western societies, slenderness is generally associated with happiness, success, youthfulness and social acceptability. Slimness is seen as a desirable attribute for women in prosperous Western cultures and is associated with self-control, elegance, social attractiveness and youth ${ }^{[27,28]}$. The ideal female shape is epitomized in the slim but full-breasted figures of models such as Elle MacPherson and Claudia Schiffer, the body type that Marchessault ${ }^{[29]}$ describes as "the physically impossible, tall, thin and busty Barbie-doll stereotype". Muscle tone is also important and the 2000s ideal is a firm-looking body for women as well as men ${ }^{[28]}$, although visible muscles are not usually considered gender appropriate for women ${ }^{[30]}$. More recently, media have tremendous effects on people's live. Several studies have shown that girls and women who have higher levels of exposure to media imagery tend to be less satisfied with the way that they look than those with lower levels of exposure ${ }^{[12,31-33]}$. Botta $^{[34]}$ found that magazine exposure (mediated by social comparison processes) was linked to body dissatisfaction in boys as well as girls and in 2003 argued that: magazine reading, social comparisons and critical body image processing are important predictors of body image and eating disturbances in adolescent boys and girls ${ }^{[35]}$.

There is also some evidence that exposure to particular kinds of television images may influence body satisfaction in girls. Although number of hours of television watched has not generally been found to correlate with body dissatisfaction, the content of what is watched has been found to predict body satisfaction and drive for thinness in girls. Women compared their bodies explicitly with those of other women (models, friends and family members) and often found their bodies wanting, leading to lowered body satisfaction. 
High self-concept, resistance to internalization of the thin ideal and perceptions of body mastery and control predict body satisfaction.

Interventions designed to promote satisfaction seem to be promising, especially those stressing body mastery and control and positive physical selfperception. Moderate exercise, focusing on body mastery and enjoyment rather than appearance and weight, may also be an effective strategy for improving body satisfaction. Cultural changes in the acceptability of a variety of body types for both men and women and a focus on body function rather than aesthetics, would be likely to improve body satisfaction for both women and men.

\section{REFERENCES}

1. Probst, M., W. Vandereycken and H. Van Coppenolle, 1998. The significance of body size estimation in eating disorders: its relationship with clinical and psychological variables. Int. J. Eat. Disord., 24: 167-174. DOI: 10.1002/(SICI)1098108X(199809)24:2<167::AID-EAT6>3.0.CO;2-C

2. King, N., S. Touyz and M. Charles, 2000. The effect of body dissatisfaction on women's perceptions of female celebrities. Int. J. Eat. Disord., 27: 341-347. http://www3.interscience.wiley.com/journal/70002 615/abstract

3. Ogedn, J. and J. Steward, 2000. The role of mother-daughter relationship in explaining weight concern. Int. J. Eat. Disord., 28: 78-83. DOI: 10.1002/(SICI)1098-108X(200007)28:1<78::AIDEAT9>3.0.CO;2-N

4. Markula, P., 1995. Firm but shapely, fit but sexy, strong but thin: The postmodern aerobicizing female bodies. Sociol. Sport J., 12: 424-453. http://www.getcited.org/pub/103336130

5. Cash, T.F., 2002. Cognitive-Behavioral Perspectives on Body Image. In: Body Image: A handbook of Theory, Research and Clinical Practice, Cash, T.F. and T. Pruzinsky (Eds.). Guilford, New York, ISBN: 10: 1593850158, pp: 530.

6. Cash, T.F., 2004. Body image: Past, present and future. Body Image Int. J., 1: 1-5. DOI: 10.1016/S1740-1445(03)00011-1

7. Roche, L.A. and H.W. Marsh, 2000. Multiple dimensions of university teacher self-concept. Instruct. $\quad$ Sci., 28: 439-468. DOI: 10.1023/A:1026576404113

8. Turner, B.S., 1992. Regulating Bodies: Essays in medical sociology. Routledge, London, ISBN: 10: 0415069637, pp: 280.
9. Feingold, A. and R. Mazzella, 2002. Gender differences in body image are increasing. Psychol. Sci., 9: 190-195. DOI: 10.1111/1467-9280.00036

10. Hagger, M.S., S.J.H. Biddle, E.W.N. Chow, N. Stambulova and M. Kavussanu. 2003. Physical self-perceptions in adolescence: Generalizability of a hierarchical multidimensional model across three cultures. J. Cross Cult. Psychol., 34: 611-642. DOI: 10.1177/0022022103255437

11. Canpolat, B.I., S. Orsel, A. Akdemir and M.H. Ozbay, 2005. The relationship between dieting and body image, body ideal, self-perception and body mass index in Turkish adolescents. Int. J. Eat. Disord., 37: 150-155. http://www.ncbi.nlm.nih.gov/pubmed/15732069

12. Anderson, D.R., A.C. Huston, K.L. Schmitt, D.L. Linebarger and J.C. Wright, 2001. Early childhood television viewing and adolescent behavior: The recontact study. Monograps Soc. Res. Child Dev., 66: 1-146. http://www.jstor.org/pss/3181552

13. Tiggemann, M. and F. Golder, 2006. Tattooing: An expression of uniqueness in the appearance domain. Body Image Int. J. Res., 3: 309-16. DOI: 10.1016/j.bodyim.2006.09.002

14. Paxton, S.J., D. Neumark-Sztainer, P.J. Hannon and M.E. Eisenberg, 2006. Body dissatisfaction prospectively predicts depressive mood and low self-esteem in adolescent girls and boys. J. Clin. Child Adolescent Psychol., 35: 539-549. DOI: 10.1207/s15374424jccp3504_5

15. Connors, J. and P. Casey, 2006. Sex, body-esteem and self-esteem. Psychol. Rep., 98: 699-704. DOI: 10.2466/PR0.98.3.699

16. Grogan, S., R. Evans, S. Wright and G. Hunter, 2004. Femininity and muscularity: Accounts of seven women bodybuilders J. Gender Stud., 13: 49-63. DOI: 10.1080/0958923032000184970

17. Shavelson, R.J., J.J. Hubner and G.C. Stanton, 1976. Self-concept: Validation of construct interpretations. Rev. Educ. Res., 46: 407-441. DOI: 10.3102/00346543046003407

18. Fox, K. and C. Corbin, 1989. The physical selfperception profile: Development and preliminary validation. $\quad$ JSEP., $\quad 1$ : 408-430. http://www.humankinetics.com/jsep/viewarticle.cf $\mathrm{m}$ ?aid $=9252$

19. Stunkard, A.J., T. Sorenson and F. Schulsinger, 1983. Use of the Danish Adoption Register for the study of Obesity and Thinness. In: The Genetics of Neurological and Psychiatric Disorders, Kety, S., (Eds.), Raven, New York. ISBN: 9780890046265. pp: 300. 
20. Fallon, A. and P. Rozin, 1985. Sex differences in perceptions of desirable body shape. J. Abnorm. Psychol., 94: 102-105. PMID: 3980849

21. Crawford, S. and R.C. Ecklund, 1994. Social Physique anxiety, reasons for exercise and attitudes toward exercise settings. JSEP., 16: 70-82. http://www.humankinetics.com/jsep/viewarticle.cf m?aid $=9028$

22. Hallinan, C. and P. Schuler, 1993. Body-shape perceptions of elderly women exercisers and nonexercisers. Percept. Mot. Skills, 77: 451-456. PMID: 8247665

23. Secord, P. and S. Jourard, 1953. The appraisal of body-cathexis: Body-cathexis and the self. J. Consult. Psychol., 17: 342-347. PMID: 13109086

24. Thompson, J. and K. Psaltis, 1988. Multiple aspects and correlates of body figure ratings: A replication and extension of fallon and rozin. Int. J. Eat. Disord., 7: 813-817. DOI: 10.1002/1098108X(198811)7:6<813::AID-

EAT2260070612>3.0.CO;2-4

25. Fingeret, C.M., D.H. Gleaves and C.A. Pearson, 2004. On the methodology of body image assessment: The use of figural scales to evaluate body dissatisfaction and the ideal body standards of women. Body Image Int. J. Res., 1: 207-212. DOI:10.1016/j.bodyim.2004.01.003

26. Thompson, J.K., L. Heinberg, M. Altabe and S.T. Dunn, 1999. Exacting Beauty: Theory, Assessment and Treatment of Body Image Disturbance. American Psychological Association, Washington, DC., ISBN: 10: 1557989877.

27. Orbach, S., 1993. Hunger Strike: The Anorectic's Struggle as a Metaphor for Our Age. Penguin, London, ISBN: 0380703939.
28. Bordo, S., 2003. Unbearable Weight: Feminism, Western Culture and the Body. University of California Press, Berkeley, ISBN-10: 0520088832.

29. Marchessault, G., 2000. One Mother and Daughter Approach to Resisting Weight Preoccupation. In: Women's Bodies, Women's Lives, Miedema, B., J.M. Stoppard and V. Anderson (Eds.). Sumach Press, Toronto, ISBN: 10: 0415165601, pp: 121.

30. Choi, P.Y.L., 2000. Femininity and the Physically Active Woman. Routledge, London, ISBN: 10: 041516561X.

31. Levine, M.P., L. Smolak and H. Hayden, 1994. The relation of sociocultural factors to eating attitudes and behaviors among middle school girls. J. Early Adolescent, 14: 471-490. DOI: 10.1177/0272431694014004004

32. Harrison, K., 2000. Television viewing, fat stereotyping, body shape standards and eating disorder symptomatology in grade school children. Commun. Res., 27: 617-640. DOI: 10.1177/009365000027005003

33. Botta, R.A., 1999. Television images and adolescent girls' body image disturbance. J. Commun., 49: 22-41. DOI: 10.1111/j.14602466.1999.tb02791.x

34. Botta, R.A., 2000. The mirror of television: A comparison of black and white adolescents' body image. J. Commun., 50: 144-159. DOI: 10.1111/j.1460-2466.2000.tb02857.x

35. Botta, R.A., 2003. For your health? The relationship between magazine reading and adolescents' body image and eating disturbances. Sex Roles, 48: 389-399. http://findarticles.com/p/articles/mi_m2294/is_200 3_May/ai_104635132 